\title{
Development Knowledge Can Increase Manufacturing Capability and Facilitate Quality by Design
}

\author{
Russ Somma
}

Published online: 14 December 2007

(C) The Author(s) 2007

The pharmaceutical industry works hard to develop, manufacture, and bring to market new drugs - and to comply with regulatory requirements to demonstrate that the drugs are safe and effective. A new approach to drug development could increase efficiencies, provide regulatory relief and flexibility, and offer important business benefits throughout the product's life cycle.

This article explores the processes used in developing a market formulation and requisite supportive data, particularly in light of the industry's current movement toward submissions based on quality by design (QbD). It outlines activities that should be performed early in the drug development process before initiating manufacturing and attempting market entry. The article identifies the type of data needed to address regulatory concerns and provides a pragmatic baseline for manufacturing facility requirements. Finally, it introduces new technologies that support the QbD approach.

The International Society of Pharmaceutical Engineering (ISPE) has undertaken an initiative [Product Quality Lifecycle Implementation (PQLI)] to clarify the requirements for implementing QbD. With this initiative, ISPE will provide a forum for presenting the current thinking within industry regarding the "how" and "what" for the science of QbD $[1,2]$.

\section{Regulatory Bodies Respond to Market Realities}

Once upon a time, a pharmaceutical company with a successful drug could count on a dozen years of peak earnings to recoup its formidable investment in the

R. Somma $(\bowtie)$

SommaTech, LLC,

Somerset, NJ, USA product's development - and to offset the costs of development efforts for products that did not make it to market. Today, rapid generation of follow-on compounds has shortened that horizon by nearly half. A first-in-class product enjoys only 7 or 8 years of peak sales before competitive products begin to erode the market. Within this narrowing window, the impact of a product recall or a product backorder is potentially enormous.

In similar circumstances, other high-tech industries have responded by reaching out to lower-cost markets for sourcing or by embracing new, more efficient, technologies. In the past, regulatory constraints may have limited the pharmaceutical industry's options. In recent years, however, the U.S. Food and Drug Administration (FDA) has launched certain initiatives designed to help relieve some of the regulatory burden on the pharmaceutical industry without sacrificing any of the quality controls so critical to patient safety. According to Janet Woodcock, M.D., director of the FDA's Center for Drug Evaluation and Research (CDER), the ultimate goal is "a maximally efficient, agile, flexible pharmaceutical manufacturing sector that reliably produces high-quality drug products without extensive regulatory oversight." [3].

To that end, FDA reevaluated its regulatory framework with an eye toward the following:

- Encouraging early adoption of new technological advances

- Facilitating adoption of modern quality management techniques

- Encouraging implementation of risk-based approaches focusing on critical areas

- Ensuring that regulatory review, compliance and inspection policies are based on state-of-the-art pharmaceutical science

- Enhancing consistency and coordination of FDA's drug quality regulatory programs 
FDA issued a final report on its Pharmaceutical Current Good Manufacturing Practices (cGMPs) for the 21st Century initiative in September 2004 [4-6]. In January 2006, the Office of Generic Drugs (OGD) announced its intention to utilize question-based review (QbR; 7). Similar reevaluations took place in Europe. In 2003, the International Council for Harmonization ( $\mathrm{ICH}$ ) announced its new vision and quality strategy; in November 2005, it issued guidelines Q8 on pharmaceutical development, Q9 on quality risk management and Q10 for the use of Quality Management as an enabler for utilization of QbD as set out in Q8 and Q9. More recently, FDA issued the same concepts in its Guidance for Industry, Q8 Pharmaceutical Development May 2006 and Q9 Risk Management June 2006 [8].

The regulatory bodies see a need for change. Industry opinion on these initiatives has been mixed. How, then, does the pharmaceutical industry move forward?

The answer lies in applying the QbD philosophy to product and process design (e.g., Q8, Q9) and establishing quality systems (e.g., Q10) that support QbD. To see the context in which this can be accomplished, it is necessary to examine the development process from end to end [9].

\section{Improvement Starts with Eliminating Silos}

Elements of the drug development process include discovery and formulation, preclinical evaluation, clinical evaluation (phases I-IV) and post-marketing or life-cycle management. Many organizations treat these components as distinct elements when, in fact, they are all stages in a continuum, each building on the previous. What's more, each of these steps represents an opportunity to build the knowledge base [10].

Drugs can be discovered in a number of ways - ranging from sheer luck to sophisticated high-throughput screening of promising molecules. Researchers reading historical records found references to the Bachelor's Button (Corn Flower /Corn Cockle); an alkaloid extracted from that flower is used in the treatment of leukemia. Some of today's research focuses on designing drugs for a disease in a specific population through molecular modeling, biotechnology, or other means. Other quests involve deep-sea bioprospecting to seek high-potential compounds - some of which are in late stages of review by regulatory authorities [11].

Although new candidates can enter the pipeline by several routes, not many make it out. Only about one in 10,000 compounds make it through the preclinical evaluation that is designed to assess what works and what is safe. The compound must undergo both in vitro and in vivo testing to assess primary safety, biological activity and therapeutic level. It often takes 3 to 4 years to develop a candidate suitable for the next step.
Phase I clinical trials, or first-in-human (FIH) studies, are small trials, usually involving from 20 to 100 normal, healthy volunteers. Over the course of a year or so, studies are conducted to determine safety in humans and dose range and route of administration. Two out of three drugs make it through Phase I. Even fewer candidates - only one in three-make it through Phase II clinical trials involving groups of 100 to 500 volunteer patients. These studies evaluate effectiveness of the compound, determine adverse events that may be associated with the compound, and select a target dose or range.

Phase III clinical trials are used to verify effectiveness and long-term safety and to establish the optimal dosage or range. This pivotal study phase involves larger numbers of volunteer patients - typically 1,000 to 3,000. A compound spends about 3 years in pivotal trials. Four out of five drugs that enter Phase III trials pass and a New Drug Application (NDA) is submitted [12].

In all, a sponsor will spend 4 to 10 years and approximately $\$ 930$ million to 1.041 billion, from the time it identifies a lead compound until the new drug is approved, manufactured, and marketed [13]. From lead identification through toxicity testing to the document preparation and regulatory interactions that go along with pivotal trials and submission, a huge knowledge base is generated. Too often, that knowledge capital is left in the laboratory or in the manufacturing facility and wasted. Captured correctly, that knowledge can work to the drug developer's advantage. To work smarter, the industry must recognize knowledge opportunities. These opportunities may present themselves in the most mundane activities such as sample weighing. Many times, key insight into a drug compound's handling and physical characteristics are revealed early on in the development process. There can no longer be operations, which are perceived as simply mechanistic operations with no value content to be added to the collective knowledge base. Every opportunity must be leveraged with the future goal of being a successful marketable product. To be useful in the QbD approach, data in the knowledge library must provide an understanding of the product and process in terms of fundamental, mechanistic properties. These data define the design space - the quality attributes of the product [14, 15].

\section{Better Science Offers Business Advantages}

Quality by Design is a systematic approach to achieving desirable quality by carefully evaluating all attributes that characterize quality, from the earliest stages of development and through out the product lifecycle. Aircraft manufacturers have used such an approach for years, documenting their design process for FAA approval. Within an approved process, the manufacturer has leeway to make changes as 
long as the changes do not result in changes to the design space [16]. For the pharmaceutical industry, QbD is a means of assuring the identity, purity, quality, and potency of a drug as it relates to its efficacy. In practical terms, it means that the product's critical quality attributes (CQAs) and process critical control points (PCCPs) must be identified and characterized in the NDA. This more robust NDA, based in solid science, can be leveraged for greater business flexibility. In a QbD environment, process or product changes that do not affect product quality could potentially be made without post-approval submission [17, 18].

The design space for a drug product encompasses variability of the product formulation, manufacturing process, manufacturing operating parameters, and raw material quality. Within that region, the control space addresses process operating parameters and raw material quality. A QbD-based drug development process identifies attributes-specifications or numerical limits that set criteria to which a product should conform to be acceptable. It further identifies critical quality attributes, or characteristics that must be controlled within defined limits to ensure acceptable product quality. QbD also recognizes that some variability is permissible and sets a range of attributes or critical process parameters that still allow for acceptable product quality and performance. Beyond those ranges lies the edge of failure where predictions of product performance are less certain. (See Fig. 1).
A successful move to $\mathrm{QbD}$ requires thinking farther ahead than is often the case today, and deploying more resources at an earlier stage in development. It also requires taking advantage of technologies that support better knowledge management. Consider the challenges involved in moving tablet development from laboratory scale to pilot or commercial scale. This change of scale traditionally has many unknowns. New technologies are available, however, that can reduce these unknowns. For example, compressionsimulation equipment make it possible to obtain explicit data about how a product runs at high speed using just a few grams, replacing the tacit knowledge of scale-up with explicit knowledge gained by using a measurable and reproducible database [19-22].

Too often, the process of defining facility needs is focused more on the present than the future. But this effort should anticipate the future, providing a clear roadmap for product development and preparing for technology transfer. Plans must address the likelihood of post-approval or life cycle changes such as increased demand. Supporting data must be maintained in meaningful summaries with narratives, defining the product, the process, and the design space [23-25].

As an industry, we already generate much of the information needed to define a design space. Throughout the development process, we obtain drug substance specifications, including physicochemical properties. We

Fig. 1 Collected data are used to refine the area of the "maximum operating range." The knowledge gained from earlier development efforts may be used to minimize risk during unexpected excursions into the "zone of potential failure"

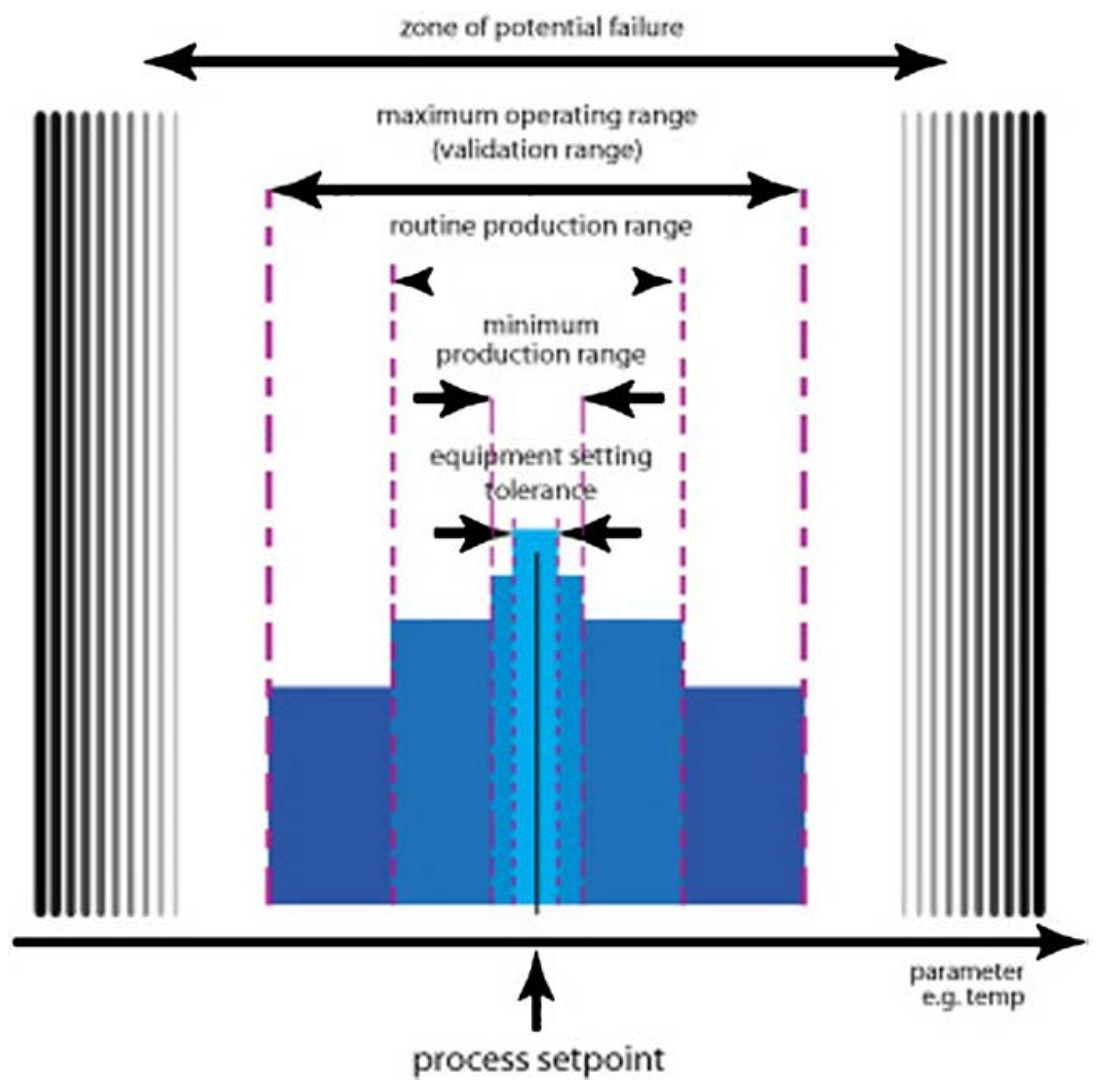


have drug product specifications and basic knowledge of excipient interactions. We understand the characteristics and variability of the raw materials. We know the desired state of our product profile and the stability of clinical forms and prototypes of the product and the drug substance. From this encyclopedia of knowledge, we can draw the data necessary to support a science-based submissionspecifically, quality attributes and critical process parameters, along with their acceptance criteria and ranges, and a manufacturing control strategy. In return, we can negotiate greater freedom to make post-approval changes or modifications that remain within the agreed-upon design space. For example, a submission provides data showing that a substance is stable at $50^{\circ} \mathrm{C}$, with a range of $\pm 10^{\circ}$. If online testing indicates that the product is not dry enough and it becomes necessary to raise the temperature, it would be possible to make the change without impacting the review $[26,27]$.

\section{Planning for Production Early in Development}

Ideally, CMC considerations begin in the earliest stages of drug development. Much knowledge is generated in the lab that may have significant bearing on production. It is important to know what properties of the drug substance impact product performance. Given the drug substance's properties, is the formulation intended to provide immediate release, improve palatability, protect the API from degradation, protect the digestive tract, etc.? Does the drug substance or the drug product have any special requirements-for example, a storage temperature range? What are the critical process steps and the parameters for each of those steps? How are the process steps monitored and controlled? Answering these questions early in the development process can help position the manufacturer for success.
Answering these questions to support the NDA and prepare for a rapid plant introduction requires establishing proven acceptable ranges and determining the impact of each process step on product quality. PAT methods can be used to an advantage in a $\mathrm{QbD}$ effort to generate the necessary data for optimizing a process and for the development of statistical process control (SPC) systems for controlling a process to achieve product quality [28].

Process development efforts, starting early in the development cycle, should be used as a platform to establish proven acceptable ranges. This will provide the foundation of a historical database for the product. The ranges may be broad during this early cycle and subsequently tightened as the development process generates more data. Building this database requires a systematic reporting method, which is referenced during production of clinical batches, scale-up and validation. Ultimately, the data becomes part of the knowledge store for the product and the basis for SPC, facility design, and maintenance ([29]; see, Fig. 2).

$\mathrm{QbD}$ requires the identification and description of all process steps, controllable parameters, and the anticipated result for exceeding the proven acceptable range (evaluating whether the risk of exceeding the range is major or minor). The operating range to be used in the plant for process control should be established based on this information. The proven acceptable range should be documented in the development and validation reports and referenced in protocols and batch records.

It is also necessary to rate the impact of each process step on product quality. This effort helps minimize the subsequent validation effort. The approach to this task must be based in both good science and common sense. The key to success lies in examining every detail that might impact product quality, from the instructions given to the personnel who weigh the raw materials to the
Fig. 2 This graphic suggests the potential nodes at which the development process may be subject to a quality risk analysis in a manner to refine the parameters for the process leading up to product and process introduction and subsequent validation

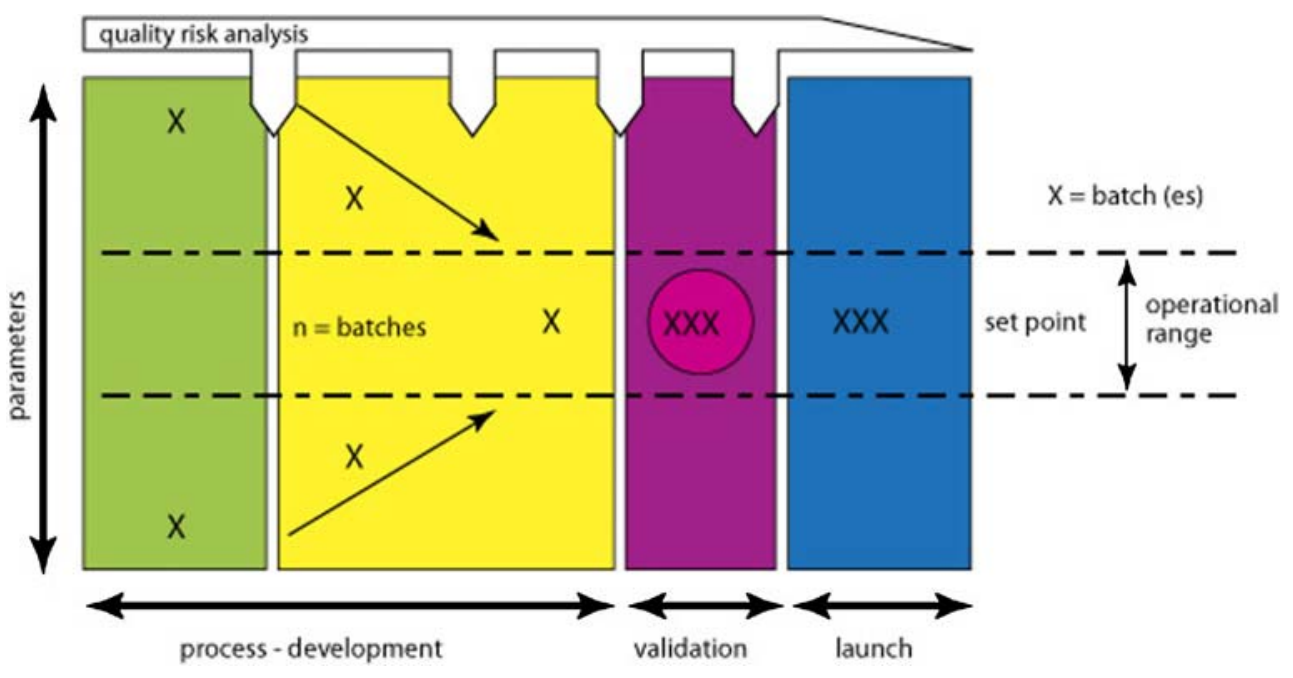


amount of material lost on drying, from the feed rate during particle size reduction to the spray rate used during coating. Capturing these data and rating the impact allows for subsequent review for atypical behavior and suspected process excursions. For documentation, the data may be shown graphically to identify process variability within established specifications. Using standardized scale-up and post-approval change (SUPAC) terms to describe this information adds clarity. With this information documented and readily available, a power outage that interrupts the process or a spike in temperature do not necessarily prevent batch release, as it would in the absence of such data (assuming that higher temperatures and process interruptions, and their impact, were documented during development).

\section{Putting Technology to Work in the Proper Context}

Process analytical technology (PAT) can be used to define the process critical control parameters (the process variables that can be controlled to maintain critical product quality attributes). We can define the requirements for parametric release of the product based on all process parameters being within pre-validated tolerances, rather than on the results of final product testing. We can also conduct systematic sensitivity analysis to determine the impact of process deviations on the product's quality attributes. PAT tools can be used for process and endpoint monitoring and control and for continuous improvement. Appropriately developed, PAT tools can be used to generate mathematical relationships promoting process understanding. Process monitoring and control tools enable real-time monitoring and, ultimately, real-time release.

It is important to remember that PAT is a tool, not a cure. PAT sensors retrofitted to an existing process will not provide the desired result unless appropriate experiments are used to develop the process understanding that is the heart of QbD. Quite often, the attempt to gain process understanding is rooted in anecdotal evidence, rather than sound scientific data. Unless developed with the appropriate scientific method, PAT systems may be more likely to ensure consistently bad product than to provide an optimized process that can be maintained through a continuous improvement philosophy. The knowledge management approach is a critical foundation. The explicit knowledge gained during development experiments forms the basis of a data set, which may be applied for process control in real time with responses based on the defined design space. Newer multivariate analytical methods and associated multivariate calibration techniques often provide much more effective PAT systems than the traditional techniques employing unidimensional analysis alone [26].
Whether using PAT or not, with knowledge management exercises, we establish a means to facilitate process understanding. With these methods, we know the critical sources of variability and can manage them. We can predict product quality attributes, and we can support our rationale for changes in output, such as batch size. It enables the riskbased approach that the FDA seeks in submissions, where the level of process knowledge is commensurate with the amount of risk to the product. Knowledge management also facilitates the definition of facility requirements, an integrated systems approach to manufacturing, and real-time product release [30].

\section{Using Knowledge Management to Work Smarter}

During drug development, we generate knowledge that falls into several categories. As a result of ongoing activities, we gain incremental knowledge, which grows with each development project. We also gain tacit or "sticky" knowledgea "feel" for the process - which we cannot communicate in a formal, systematic, or codified language. It is critical to turn such tacit knowledge into explicit knowledge, which we can set down in procedures and codify easily.

Explicit knowledge is the basis of our work. We can use explicit knowledge to develop robust formulations, meaningful specifications, and provide more flexibility during facility design than would be available using a less science-/datadriven approach. Explicit knowledge is quantifiable and easily transferable; it can be a cost-effective tool for improving our processes. When we manage explicit knowledge appropriately, it produces a well-defined set of core technologies, and it speeds development and process introduction.

Incremental knowledge provides momentum for rethinking business processes and plays a key role in continuous improvement. We learn as we go and share the experience. Sharing incremental knowledge through handbooks and other means such as SOPs moves the organization's collective knowledge base forward. For pharmaceutical manufacturing, having information available reduces uncertainty, which, in turn, accelerates process transfer from lab to pilot and on up the scale, eliminating the need to over-design the facility.

Today's sophisticated data management tools can help us gather, store, and retrieve this knowledge and share itwithin teams and across teams - so that we can better put it to work in service of continuous improvement of our processes, our facilities, and our business.

Open Access This article is distributed under the terms of the Creative Commons Attribution Noncommercial License which permits any noncommercial use, distribution, and reproduction in any medium, provided the original author(s) and source are credited. 


\section{References}

1. Roth G. Engineering and quality: quality guidelines. Contract Pharma Jan/Feb 2007.

2. Draft PQLI Summary Update Report V04, September 17,2007, ISPE PQLI Task Team.

3. Woodcock J. Pharmaceutical quality in the 21 st Century-an integrated systems approach, December 6, 2006.

4. Joshi Y, LoBrutto R, Serajuddin ATM. Industry opinion of regulatory influence: the initiative on pharmaceutical cGMPs for the 21 st century. Journal of Process Analytical Technology July/ August 2006.

5. Nasr MM. A New Pharmaceutical Quality Assessment System (PQAS) for the 21st Century, AAPS Workshop October 2005.

6. QbD Principles to be implemented in future FDA guidance; DIA Dispatch, Oct. 28, 2005.

7. Yu, LX. Implementation of quality by design: question Based Review, DIA Meeting, June 2006.

8. Chen C. Implementation of ICH Q8 and QbD-an FDA Perspective, PharmaForum Yokohama, June 2006.

9. Gassmann O, von Zedtwitz M. Organization of industrial R\&D on a global scale. R\&D Management 1998;28(3):147-61.

10. Berridge JC. Quality by design: integration of prior knowledge and pharmaceutical development into CMC submission and review: an Industry Perspective. AAPS Workshop, October 2005.

11. Krajick K. Medicine from the sea. Smithsonian Magazine May 2004.

12. Hussain AS. Quality by design: Integration of prior knowledge and pharmaceutical development into $\mathrm{CMC}$ submission and review: FDA Perspective, AAPS Workshop, October 2005.

13. Parexel's bio/pharmaceutical statistical sourcebook 2007/2008 Editor Mathieu M.P. Parexel International Corp. 2007.

14. Design space to facilitate quality initiative implementation. The Gold Sheet, April 2005.

15. DePalma A. Pharma embraces quality by design. Pharm Manuf September 2006.
16. The FAA and industry guide to product certification, Second Edition, September 2004.

17. Hoiberg C. An industry perspective of how implementing the ICH Q8 guideline may impact the pharmaceutical process development, ISPE Washington Conference, June 2006.

18. Jagota N. An industry perspective on the FDA CMC pilot program. FDA Briefing Document April 2006.

19. Rangaswamy A, Lilien GL. Software tools for new product development. J Mark Res 1997;34(1):177-84.

20. Subramanian M, Rosenthal SR. Global new product development processes: Preliminary findings and research propositions. J Manag Stud 1998;35(6):773-96.

21. Werther WB, Berman E, Vasconcellos E. The future of technology management. Organ Dyn 1994;22(3):20-32.

22. Celik M. Overview of compaction data analysis techniques Drug Dev Ind Pharm 1992;18(6\&7):767-810.

23. Castells X, Hu Y, Stewart B, Geoffroy J. Application of quality by design knowledge from site transfers to commercial operations already in progress. Journal of Process Analytical Technology January/February 2006.

24. Popp KF. Organizing technology transfer from research to production. Drug Dev Ind Pharm 1987;13(13):2339-62.

25. Von Doehren PJ, Saint John Forbes F, Shively CD. Approach to the characterization and technology transfer of solid dosage form processes Pharm Technol 1982;6:139-56 Sept.

26. McCready C. Multivariate analysis for manufacturing quality systems. Pharma Manufacturing October 2005.

27. Wechsler J. Regulatory beat: Quality standards to reshape manufacturing. BioPharma International February 1, 2007.

28. Kieffer R, Torbeck L. Validation and process capability. Pharm Technol 1998;22(6):66-76.

29. PQRI working group. Process Robustness-A PQRI White Paper. Pharm Eng November/December 2006.

30. Bush L. The end of process validation as we know it? Pharm Technol August 2, 2005, p. 36. 